\title{
Inländisches Fachkräftepotential in Spitälern, Kliniken und Pflegeinstitutionen
}

\author{
Das Gesundheitswesen leidet unter akutem Fachkräftemangel und muss deshalb mehr inländische Fach- \\ kräfte mobilisieren. Die Annahme der Volksinitiative «Gegen Masseneinwanderung» verschärft die Situation \\ zusätzlich.
}

\section{David Schürch ${ }^{a}$, \\ Jürg Winkler ${ }^{b}$}

a Master PMP, Praktikant Politik bei H+ Die Spitäler der Schweiz

b H+ Die Spitäler der Schweiz, Projektleiter Personal- und Bildungspolitik
Korrespondenz: David Schürch Jürg Winkler (ab 20.1.2015) $\mathrm{H}+$ Die Spitäler der Schweiz Lorrainestrasse $4 \mathrm{~A}$ CH-3013 Bern Tel. 0313351135 Fax 0313351170

\section{Verschärfter Fachkräftemangel}

Patienten in Schweizer Spitälern, Kliniken und Pflegeinstitutionen dürfen sich glücklich schätzen, denn das Schweizerische Gesundheitssystem gehört zu den qualitativ besten der Welt. Kaum ein anderes Land verfügt über eine so hohe Dichte an Pflegepersonal pro Patient(in) wie die Schweiz. Das gleiche gilt für die Ärzte und das medizinisch technische und therapeutische Personal. Doch aufgrund demographisch bedingter Alterung, des Rückgangs der arbeitenden Bevölkerung und der Zunahme anspruchsvoller Patienten leiden Spitäler, Kliniken und Pflegeinstitutionen am akuten Fachkräftemangel.

Die am 9. Februar 2014 vom Schweizer Stimmvolk angenommene Volksinitiative «Gegen Masseneinwanderung» wirkt sich negativ auf Spitäler, Kliniken und Pflegeinstitutionen aus. Bereits heute gelingt es den Spitälern, Kliniken und Pflegeinstitutionen nicht, den Fachkräftebedarf zu decken. Die Initiative will die Zuwanderung beschränken und durch eine gesteigerte Rekrutierung von inländischen Arbeitskräften den Rückgang der Zuwanderung kompensieren. Eine restriktive Umsetzung des Verfassungsartikels 121 a würde den Fachkräftemangel zusätzlich verstärken.

\section{Ausländisches Pflegepersonal in Spitälern, Kliniken und Pflegeinstitutionen} Gemäss Bundesamt für Statistik arbeiten in Schweizer Spitälern, Kliniken und Pflegeinstitutionen 2013 rund 185000 Personen. 32\% oder 59000 davon sind ausländischer Herkunft. Aktuelle Beobachtungen und Schätzungen zeigen, dass auch ausländisches Personal von demographischen Entwicklungen und einer durchschnittlichen, jährlichen Dropout- und Fluktuationsrate von ca. 16\% betroffen ist. Spitäler, Kliniken und Pflegeinstitutionen benötigen zur Schliessung dieser Lücke jährlich rund 10000 zusätzliche ausländische Gesundheitsfachkräfte.

\section{Schlummerndes Potential}

Um auf die aktuell auf Spitäler, Kliniken und Pflegeinstitutionen einwirkenden soziopolitischen Herausforderungen $\mathrm{zu}$ reagieren, schätzte $\mathrm{H}+$, der

\section{Potentiel de main d'œuvre indi- gène pour le secteur de la santé}

La pénurie aiguë de spécialistes que l'on connaît actuellement met en péril la qualité élevée des soins de notre pays. L'initiative populaire «Contre l'immigration de masse» du 9 février 2014 conduira à limiter l'immigration, et ainsi à aggraver la situation. Les hôpitaux doivent donc agir de toute urgence et mobiliser davantage de main d'œuvre indigène. Sur les 185000 salariés des hôpitaux, cliniques et institutions de soins, $32 \%$ sont d'origine étrangère. Selon les estimations actuelles, nous aurons besoin à l'avenir d'environ 10000 spécialistes étrangers supplémentaires chaque année. D'après l'Office fédéral de la statistique, 6,8 millions d'habitants étaient aptes au travail en 2013, dont 204000 étaient sans emploi. Si l'on y ajoute les 904000 personnes qui ne font pas partie de la population active, la main d'œuvre potentielle se monte à 1,108 million de personnes. Le secteur hospitalier a cependant besoin de spécialistes formés et expérimentés, disponibles immédiatement. Compte tenu des critères spécifiques à la profession et au secteur, $\mathrm{H}+$ évalue le potentiel pour les hôpitaux entre 1000 et $\mathbf{3 0 0 0}$ personnes, même si les modalités du recours unique à ce potentiel ne sont pas encore définies et que celui-ci ne permettra pas de couvrir le besoin annuel.

Schweizerische Spitzenverband der Spitäler, Kliniken und Pflegeinstitutionen, mittels fakten- und erfahrungsbasierten Überlegungen das Potential an inländischen Fachkräften ab.

Gemäss Bundesamt für Statistik lebten 2013 in der Schweiz 5,4 Millionen Menschen, die zwischen 
15 und 64 Jahre alt sind und somit unter die erwerbsfähige Bevölkerung fallen. Davon sind 4,46 Millionen erwerbstätig und 204000 erwerbslos. In den 204000 Erwerbslosen und 904000 Nichterwerbspersonen (Hausfrauen/Hausmänner und Personen in Ausbildung) schlummert ein inländisches Arbeitskräftepotential von 1,108 Millionen. Spitäler, Kliniken und Pflegeinstitutionen sind jedoch nur ein kleiner Teil der Gesamtwirtschaft und benötigt ausgebildete Fachkräfte mit Gesundheitsberufen und Praxiserfahrung. Die folgenden Faktoren brechen das ursprüngliche Brutto-Potential auf das Netto-Potential für Spitäler, Kliniken und Pflegeinstitutionen herunter: gruppen. Die rund 185000 Erwerbstätigen der Spitäler, Kliniken und Pflegeinstitutionen stehen gemäss Krankenhausstatistik 2013 des Bundesamts für Statistik zur Gesamtzahl Erwerbstätiger in der Schweiz von 4,65 Millionen im Verhältnis von 1:25 zueinander. Das heisst, dass eine von 25 Personen in der Schweiz in einem Spital, einer Klinik oder Pflegeinstitution arbeitet. Der Einfachheit halber und mit der Absicht, keine Branche zu diskriminieren, wird angenommen, dass sich das Inländerpotential proportional zur Beschäftigtenzahl auf alle Sektoren, Branchen und Berufsgruppen gleichmässig verteilt. Für die Spitäler, Kliniken und Pflegeinstitutionen wären das dann $371000 \cdot 1 / 25=14840$ Personen.

\section{«Schweizer Spitäler, Kliniken und Pflegeinstitutionen sind weiterhin auf die ungehinderte Zuwanderung ausländischer Arbeitskräfte angewiesen.»}

\section{Alterskategorie}

Von den 1,108 Millionen betrachten wir nur die Alterskategorien der 25- bis 54-Jährigen. Das ist eine Modellannahme und begründet sich wie folgt: $\mathrm{Ab}$ 25 ist die Zielgruppe fertig ausgebildet, hat bereits etwas Berufserfahrung und ist somit sofort einsetzbar. Die Obergrenze von 54 setzen wir dort, wo wir heute einen vermehrter Berufsausstieg (Dropouts) beobachten und es für Nichterwerbstätige alters- und gesundheitsbedingt schwierig wird, sich in den belastenden Berufsalltag einzugliedern.

\section{Ausbildungsstufe}

Zur Bekämpfung des Fachkräftemangels brauchen Spitäler, Kliniken und Pflegeinstitutionen zweckmässig qualifizierte Arbeitskräfte. Der Bedarf an Ausbildungen auf Sekundarstufe II und Tertiärstufen A und B ist dabei besonders hoch. Es handelt sich hierbei primär um Fachpersonen Gesundheit, diplomierte Pflegefachpersonen und spezialisiertes Pflegefachpersonal. Ungelernte und Akademiker haben wir in diesen Überlegungen bewusst ausgeschlossen, da kaum Bedarf an Ungelernten vorhanden ist und die Anzahl erwerbsloser Akademiker vernachlässigbar gering ist.

Gemäss Bundesamt für Statistik waren 2013 in der Schweiz demnach nach Anwendung der zwei oben genannten Kriterien rund 371000 Personen entweder erwerbslos oder fielen unter die Kategorie Nichterwerbspersonen und verfügen über eine Ausbildung auf Sekundarstufe II oder eine Tertiärausbildung. Dieses Potential wird im nächsten Schritt weiter auf Spitäler, Kliniken und Pflegeinstitutionen reduziert.

\section{Branchenanteil}

Die oben erwähnten Inländerpotential (371000 Personen) verteilt sich auf alle Branchen und Berufs-
Dieses Bruttopotential wird in einem letzten Schritt zusätzlich verfeinert.

\section{Weitere Kriterien}

Das mögliche Potential an inländischen Fachkräften vermindert sich zusätzlich nach Anwendung von sechs technischen oder individuellen Kriterien:

- Rekrutierungsprozess: Hier scheiden ungeeignete Gesundheitsfachleute aus.

- Aus- und Weiterbildung: Es ist anzunehmen, dass Personen erneut eine Aus- oder Weiterbildung angetreten haben und für den Berufseinsatz momentan wegfallen.

- Gesundheit: einige der Personen können aus gesundheitlichen Gründen nicht in Spitälern, Kliniken und Pflegeinstitutionenarbeiten arbeiten.

\section{Inländisches Potential ist unzureichend}

Die erste, grobe Schätzung des inländischen Fachkräftepotentials durch $\mathrm{H}+$ verdeutlicht, dass im Inland ein gewisses Potential an Fachkräften vorhanden ist. Unter den aktuellen politischen und tariflichen Verhältnissen können 1000 bis höchstens 3000 Fachkräfte aus dem Inland gewonnen werden. Die Mobilisierung dieses Fachkräftepotentials selbst bedeutet finanziellen und organisatorischen Mehraufwand. Eine ernüchternde Tatsache ist, dass dieses Potential lediglich einmalig ausgeschöpft werden kann und nicht einmal ein Drittel der benötigten Fachkräfte eines Jahres deckt. Schweizer Spitäler, Kliniken und Pflegeinstitutionen sind deshalb weiterhin auf die ungehinderte Zuwanderung ausländischer Arbeitskräfte angewiesen. 
Abbildung 1

Inländisches Fachkräftepotential in Spitälern, Kliniken und Pflegeinstitutionen (Quelle: H+ und Bundesamt für Statistik, BFS; 2014).

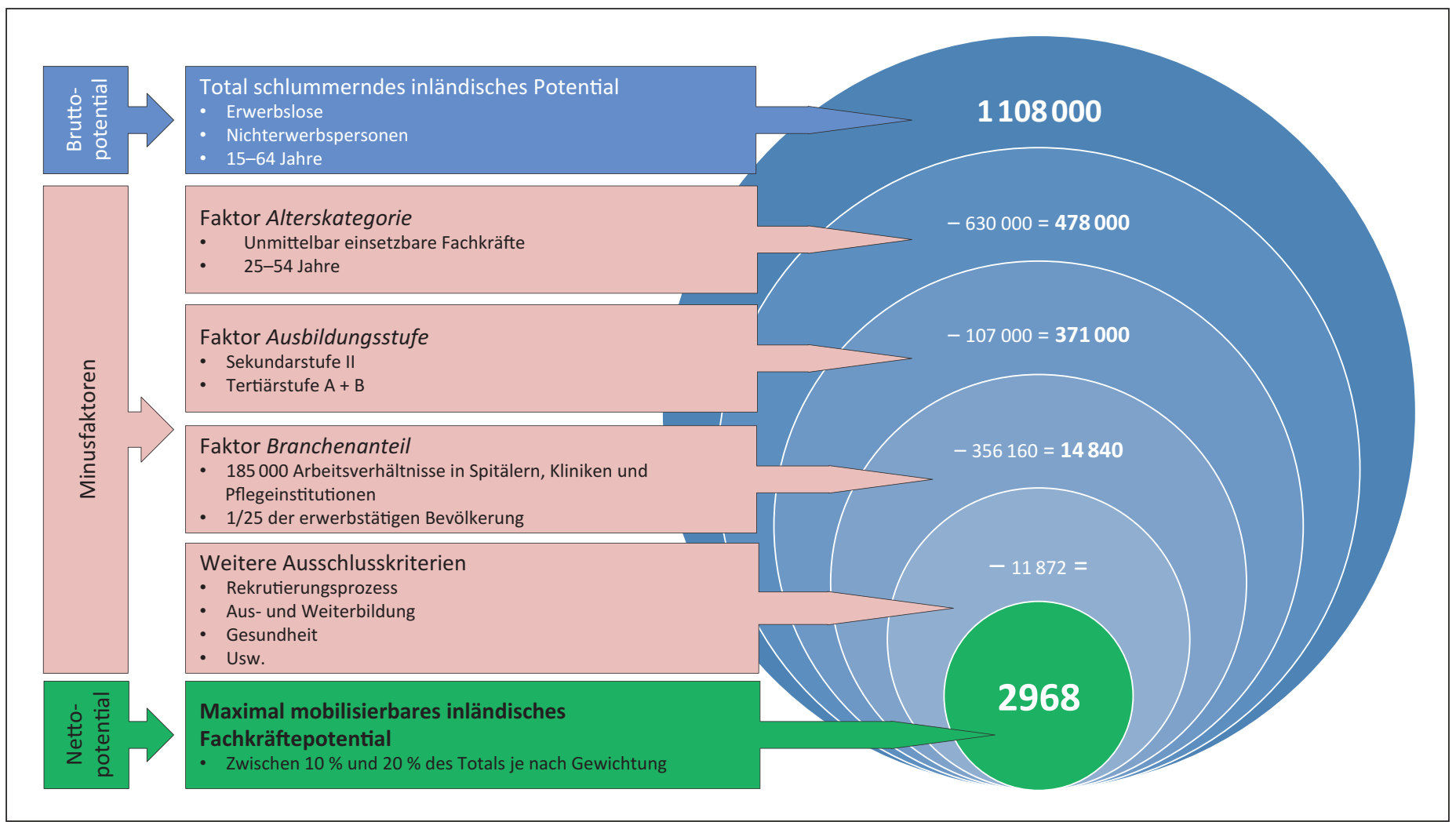

- Freiwilliger Verzicht auf Erwerbstätigkeit: Einige Gesundheitsfachpersonen nehmen eine Rolle als Hausfrau/-mann wahr und verzichten deshalb bewusst auf eine Erwerbstätigkeit.

- Andere Verwirklichung: Gesundheitsfachpersonen, die eine andere, persönlich oder beruflich bedingte Verwirklichung ausserhalb von Spitälern, Kliniken und Pflegeinstitutionen anstreben, fallen ebenfalls weg.

- Kinderpause: Gesundheitsfachleute, die eine Kinderpause einlegen, bleiben dem Arbeitsmarkt erfahrungsgemäss im Alter von 30 bis 40 Jahren während mehreren Jahren fern.

Auf der Grundlage von mehrjährigen branchenspezifischen Erfahrungen erwarten wir, dass nach Anwendung der zuvor dargelegten Kriterien mindestens 4 von 5 Personen nochmals wegfallen.

Lediglich 10\%-20\% der verbleibenden 14840 Personen können also effektiv für Spitäler, Kliniken und Pflegeinstitutionen gewonnen werden. Je nach Szenario bewegt sich dieses Potential zwischen 1484 (10\%) oder 2968 (20\%) Personen.

Die Abbildung 1 illustriert die einzelnen Schritte zur Schätzung und Berechnung des inländischen Fachkräftepotentials.

\section{Aussicht}

Mit der vorliegenden Schätzung des mobilisierbaren inländischen Fachkräftepotentials beabsichtigt $\mathrm{H}+$, erste konkrete Überlegungen aufzuzeigen, um zukünftige personelle und gesetzliche Herausforderungen anzugehen. Weitere Möglichkeiten, Fachkräfte im Inland zu gewinnen, bestünden zum Beispiel darin, bereits Teilzeit arbeitende Fachkräfte zu höheren Arbeitspensa zu motivieren. Diese und weitere Möglichkeiten, das Inländerpotential besser zu nutzen, werden in der vorliegender Berechnung bewusst nicht mitberücksichtigt, weil dafür zu wenig Zahlenmaterial vorliegt.

\section{Referenzen}

- Bundesamt für Statistik BFS 2014. Ausbildungsstufen der ständigen Wohnbevölkerung nach Arbeitsmarktstatus und Altersgruppe 2013.

\section{Articles interactifs}

Vous souhaitez commenter cet article? II vous suffit pour cela d'utiliser la fonction «Ajouter un commentaire» dans la version en ligne. Vous pouvez également consulter les remarques de vos confrères sous: www.bullmed.ch/ numero-actuel/articles-interactifs/ 